\title{
Cognitive Deficits in the Early Stages of Alzheimer's Disease
}

\author{
Martha Storandt \\ Washington University in St. Louis
}

\begin{abstract}
Evidence from longitudinal, experimental, and neuroimaging studies converge to indicate that psychological functions other than episodic memory are affected very early in the course of Alzheimer's disease and, indeed, may predate or influence the apparent memory deficits. Changes in personality and difficulty in executive function, especially in terms of attentional and inhibitory control, are especially prominent. Deficits in other types of memory (i.e., semantic memory, conditioning) can also be detected in the early stages of the disease. It is time to update existing diagnostic criteria for this form of dementia in terms of current knowledge of multiple and interacting brain systems.
\end{abstract}

KEYWORDS-dementia, executive function, attention, inhibitory control, memory systems

Alzheimer's disease (AD) is a pathological condition associated with age. Longitudinal composite scores from a cognitive test battery for two initially unaffected men of similar age and education are shown in Figure 1. Autopsies revealed that one of the men had $\mathrm{AD}$, whereas the other man had a normal brain. The longitudinal trajectory clearly shows the transition in cognitive performance and progressive downward course for the individual with AD.

Difficulty with episodic memory has become known as the hallmark symptom of AD. Families report that the person repeats questions, does not recall recent events, or forgets appointments. The traditional perspective is that the more global effects on cognition arise only after the disease progresses. There are now several converging lines of evidence indicating that other psychological functions also may be affected very early in the course of the disease and, in some people, may appear before deficits in episodic memory. This article focuses on the other psychological functions disrupted in the early stages of AD.

Address correspondence to Martha Storandt, Department of Psychology, Washington University, St. Louis, MO 63130; e-mail: mstorand@wustl.edu.

\section{THE TRANSITION FROM NORMAL AGING TO AD}

The diagnostic criteria for $\mathrm{AD}$ are shown in Box 1. One reason it has been difficult to determine whether other types of psychological deficits exist in AD before memory is affected is that memory loss is required for the diagnosis of $\mathrm{AD}$, or any other dementia, and is therefore required for admission to a study sample. Population-based longitudinal studies that begin with people who have not (yet) manifested dementia avoid this problem. In the Framingham study (Elias et al., 2000), two baseline measures were related to the diagnosis of probable $\mathrm{AD}$ 22 years later: One was verbal memory, and the other was the Similarities subtest from the Wechsler Adult Intelligence Scale, a test of abstract reasoning. In the Berlin aging study (Rapp \& Reischies, 2005), poor initial performance on measures of attention and executive function were better predictors of which nondemented individuals were diagnosed with AD 2 years later than were tests of episodic memory. Chen et al. (2005) longitudinally followed a group of people before any developed $\mathrm{AD}$ and found that the greatest prediagnosis decline observed in individuals who subsequently were diagnosed with $\mathrm{AD}$ was on a timed trail-making test involving planning and sequencing.

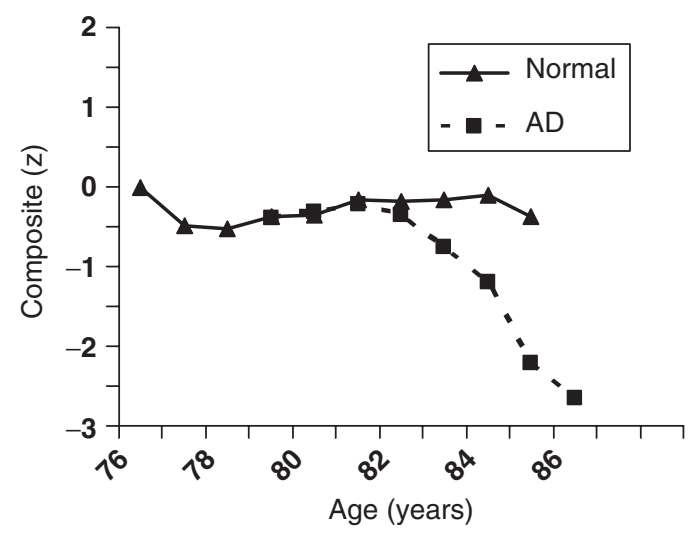

Fig. 1. Longitudinal performance on a composite of cognitive test scores by two men of similar age and education included in sample reported by Galvin et al. (2005); one had AD at autopsy, the other had a normal brain. 


\section{BOX 1}

Diagnostic Criteria for Alzheimer's-Type Dementia

A. Multiple cognitive deficits in both

1. Memory and

2. One or more of the following

a. Aphasia

b. Apraxia

c. Agnosia

d. Executive function

B. Impairment in social or occupational function that represents a decline from previous levels

C. Gradual onset and continued cognitive decline

D. Deficits not due to other causes

Abridged from American Psychiatric Association (1994, pp. 142-143).

In addition to longitudinal studies beginning with cognitively healthy older people, some studies focus on individuals who have experienced some decline but do not meet existing criteria for a diagnosis of AD. A variety of labels have been used to describe this condition. The label with greatest popularity in recent years is mild cognitive impairment (MCI), which initially focused solely on deficits in episodic memory but was later expanded to include deficits in other cognitive domains. My colleagues and I (Storandt, Grant, Miller, \& Morris, 2006) examined the longitudinal course and neuropathologic diagnoses of individuals to whom MCI criteria were applied; nearly all who died and came to autopsy were found to have AD.

More important here, however, was where these individuals began. Only $61 \%$ of those with MCI according to the expanded criteria began with memory deficits. The initial problems for the remaining $39 \%$ were visuospatial (11\%), executive function (19\%), or combined visuospatial and executive (9\%) deficits. Psychomotor slowing may play a role because the tests of visuospatial ability available for this analysis were all timed. The tests of executive function (e.g., digit span, letter fluency) did not include some of the more sophisticated measures developed since that longitudinal study began. Nevertheless, more than one third of the people who ultimately had pathologically confirmed AD began with deficits in cognitive abilities other than those typically thought of as episodic memory.

Our results (Storandt et al., 2006) also showed that we could detect deficits in cognition due to the underlying process of $\mathrm{AD}$ even before MCI, by using information about changes from a person's previous levels of function obtained from other people who knew that person. In other words, people labeled pre-MCI had declined from their own previous level of function, even though their current objective performance had not yet fallen below a cut point for MCI based on group norms.

There are also important noncognitive changes that may occur before diagnosis. In a prospective longitudinal study of older adults without dementia, approximately half of those who subsequently developed dementia had already experienced changes in personality (Balsis, Carpenter, \& Storandt, 2005), according to informant reports that occurred at least a year before diagnosis of AD. The common changes were increased rigidity, apathy, self-centeredness, or emotional lability. Similarly, in a large population-based study involving African Americans in the United States and Yoruba in Nigeria (Smith-Gamble et al., 2002), informants were asked at initial screening about personality change: People without dementia but with reported personality change were twice as likely to be diagnosed with AD 2 years later.

Thus, it is clear that psychological functions other than memory are affected quite some time before eventual diagnosis of AD. Prominent among these are personality and higher-order cognitive functions such as reasoning and executive function. Indeed, sometimes the first notable indications of the disease may be unrelated to episodic memory: Families first observe that the person has difficulty with familiar tasks such as preparing a meal, driving a car, or balancing a checkbook. Often, the tasks that are affected involve executive functions such as attention, working memory, and inhibitory control.

\section{THE ROLE OF EXECUTIVE FUNCTION}

Some of the early difficulties with executive function reported in the longitudinal studies may contribute to the better-recognized deficit in episodic memory. Balota and his colleagues suggest that much of the difficulty with episodic memory in AD may be caused by deficits in attentional mechanisms (see Balota \& Faust, 2001, for a review). Attention has many aspects, but there are at least two classes of processing deficits in AD that involve attention. One involves inhibitory control; the other involves maintenance of task sets.

Some aspects of implicit memory, such as spatial cuing, repetition priming, and semantic priming, are relatively unaffected by AD initially. These abilities involve the relatively automatic activation of existing information, which appears to be intact. In the everyday world, people with $\mathrm{AD}$ can sing, read, and write well into the later stages of the disease. The difficulty appears to arise with inhibiting this automatically activated information.

To better understand how attention is related to memory, consider a popular method used to study false memory. People study a list of words related to the word sleep: bed, night, dream, and so on. Sleep, however, is not on the list. When asked to recall the list, many people include the word sleep. This false memory occurs because of an imbalance in the strength of two sources of information: one based on actual recollection of the specific items presented, and the other based on the automatic activation of sleep through its association with the other words on the list. Through attentional control, one focuses on the recollection process of words actually presented and inhibits words brought to mind through other mechanisms (e.g., spreading activation through the semantic network). People with early-stage AD have trouble doing this. Individuals with AD show a higher proportion of false memories than do cognitively healthy older adults, even 
after adjusting for their poorer episodic memory (Balota \& Faust, 2001). This is an example of how selective attention may produce apparent memory difficulties.

A second way that attentional control is involved in the cognitive deficits observed in AD centers around maintenance of the task set and goals. Consider the Stroop color-naming task. Words printed in various colors are shown; the person is told to name the color rather than say the word. People with AD can do this when the words are unrelated to the colors (e.g., book, river). They clearly remember the instruction to name the color. In the conflict condition, however, the words are names of colors. This pits two tendencies against each other-the tendency to name the prepotent word dimension versus the task instruction to name the color dimension. People with AD have disproportionate difficulty in this condition, reflecting difficulty in maintaining the appropriate task set, the quality of the task set, or both (Balota \& Faust, 2001).

Semantic memory can also be impaired early in the course of AD. Semantic memory involves stored world knowledge and can be assessed by measures such as the Boston Naming Test, in which people look at drawings of various objects and name them. Often, people with AD cannot recall the name of the object (e.g., beaver) but can remember the name of the category to which it belongs (i.e., animal). Koenig, Smith, Moore, Glosser, and Grossman (2007) reported two studies involving categorization that sought to determine why deficits in semantic memory occur in AD. They pointed out that there are two processes that can be used to categorize objects. Again, one is relatively automatic: it depends on similarity to other exemplars of the category. For example, a betta can be classified as a fish based on its similarity to bass and goldfish. The other process is rule based and depends on executive functions, including attention and inhibitory control; this is the process used to classify a whale as a mammal, rather than as a fish. Using biologically plausible novel animals as stimuli, Koenig et al. found that people with AD performed well using the similarity process (the stimulus looked like the exemplar) but had difficulty with the rule-based process, which depends on executive functions (the stimulus had three of four required characteristics).

\section{AD, NEUROPATHOLOGY, AND COGNITION}

It is becoming increasingly clear that AD-related neuropathology can begin before impaired memory is observed. Galvin et al. (2005) found that nearly half of the individuals who appeared to be without dementia at the time of death had sufficient neuropathology to be diagnosed with AD. Thus, it is critical to identify the behavioral effects of these initial brain changes.

Amyloid plaques and neurofibrillary tangles are the hallmark neuropathological signs of AD. The extracellular amyloid plaques are hard, insoluble accumulations of a protein fragment that would be broken down and eliminated in a healthy brain. Neurofibrillary tangles are insoluble twisted fibers inside neu- rons that consist primarily of an abnormal form of a protein called tau, which, in its usual form, is part of the structure of the microtubules that form part of the cells' transportation system. The relative roles of these two abnormalities are a matter of current debate but are beyond the scope of this article.

A significant recent breakthrough in understanding the neuropathology in $\mathrm{AD}$ is the ability to measure amyloid plaque in the living brain. In the preclinical and early stages of AD, amyloid plaque burden appears to be high in the precuneus in the parietal lobes and in the medial prefrontal cortex (Mintun et al., 2006). These two areas of the brain are active during the conscious resting state and are thought to be involved in broad, continuous information gathering and evaluation. The activity in these two areas decreases when areas of the brain involved in goal-directed behavior are activated (Raichle et al., 2001). This finding is intriguing in light of the earlier arguments regarding changes in executive control. One could speculate that pathology in these areas might interfere with that deactivation process, making it difficult to turn attention to a specific task. One could interpret the pattern of results reported by Celone et al. (2006), who paired first names with novel faces in an fMRI study of an associative-memory task, in such a way. In the nondemented individuals, activity increased in the hippocampus during the memory task (the hippocampus is involved in the memory system supporting episodic memory) and decreased in the resting state network. In people with $\mathrm{AD}$, hippocampus activity did not increase, and resting network activity did not decrease. Of course, this reciprocal relation could fail because of a breakdown of either one or both of these interacting systems. Traditionally, the hippocampus system has been blamed. Mintun et al. (2006), however, found minimal plaque burden in the hippocampus in the early stages of Alzheimer's-type dementia, although it is possible that atrophy might occur without plaque deposits.

\section{MULTIPLE MEMORY SYSTEMS}

Problems with memory are still the most commonly observed first indication of the onset of $\mathrm{AD}$, and the focus is usually on episodic memory. The last 20 years have brought tremendous advances in cognitive neuroscience and the concomitant understanding of the multiple memory systems that are associated with different brain regions and how they can interact with each other (McDonald, Devan, \& Hong, 2004). In contrast to episodic memory, which largely depends on the hippocampal system, classical fear conditioning depends on pathways involving the amygdala network. One study found that classical fear conditioning was severely impaired in people in the early stages of $\mathrm{AD}$, whereas age-matched controls showed robust conditioning to the aversive noise as indicated by skin-conductance responses (Hamann, Monarch, \& Goldstein, 2002). Neuroimaging studies have similarly implicated the amygdala in AD (e.g., Basso et al., 2006). Given that the amygdala appears to be tuned to emotional 
stimuli, it may be involved in some of the behavioral changes that may predate AD diagnosis (Balsis et al., 2005).

\section{CONCLUSIONS AND FUTURE DIRECTIONS}

The current criteria for the diagnosis of $\mathrm{AD}$ require that at least one other cognitive ability be affected in addition to memory (Box 1). Undoubtedly, executive functioning (attentional control, working memory, inhibition) is impaired very early in the course of the disease and may even be a significant contributor to the apparent memory deficit. However, classical aphasia (inability to produce or comprehend speech), apraxia (inability to carry out purposeful movement), and agnosia (inability to recognize environmental stimuli but without sensory or memory impairment) are rarely seen in the early stages of AD, although they may be prominent in some of the rare atypical-onset variants (e.g., primary progressive aphasia, posterior cortical dysfunction). The diagnostic requirement of deficits in two areas of cognition may be counterproductive if the goal is to identify AD as early as possible. It is clear that $\mathrm{AD}$ can begin with a detectable deficit in only one cognitive domain and that the impaired domain does not necessarily need to be memory. Thus, it is time to reconsider the criteria for the clinical diagnosis of AD. Exclusion of those with difficulties other than memory from research studies contributes to an incomplete picture of the beginnings of $\mathrm{AD}$.

Although the common emphasis in diagnosing AD is on retrieval from episodic memory (i.e., delayed recall), the episodicmemory deficit at the very early stages of the disease more likely involves difficulty at the encoding stage (Twamley, Ropacki, \& Bondi, 2006), which is highly dependent on attentional-control systems. Future research should focus on the mechanisms by which other components of cognition contribute to the episodicmemory failures in dementia. Emphasis should also be placed on understanding how the disease process interferes with these other components of cognition. Difficulty with retrieval from semantic memory may also be apparent in AD. There is limited evidence concerning memory systems other than that involving the hippocampus, but what evidence there is suggests that fear conditioning, which is based on the amygdala network, is also compromised. Future research should be broadened to examine all memory systems, not just the system including the hippocampus.

\section{Recommended Reading}

Squire, L.R. (2004). Memory systems of the brain: A brief history and current perspective. Neurobiology of Learning and Memory, 82, 171-177. An accessible minireview of current thinking about the biological systems involved in memory.

Twamley et al. (2006). (See References). A critical review of 91 studies of cognitive function and neuroimaging in preclinical AD.

Winblad, B., Palmer, K., Kivipelto, M., Jelic, V., Fratiglioni, L., Wahlund, L.-O., et al. (2004). Mild cognitive impairment-beyond controversies, towards a consensus: Report of the International Working Group on Mild Cognitive Impairment. Journal of Internal Medicine, 256, 240-246. Expanded criteria for mild cognitive impairment.

\section{REFERENCES}

American Psychiatric Association. (1994). Diagnostic and statistical manual of mental disorders (4th ed.). Washington, DC: Author.

Balota, D.A., \& Faust, M.E. (2001). Attention in dementia of the Alzheimer type. In F. Boller \& S.F. Cappa (Eds.), Handbook of neuropsychology (2nd ed., Vol. 6, pp. 51-80). Amsterdam: Elsevier Science.

Balsis, S., Carpenter, B.D., \& Storandt, M. (2005). Personality change precedes clinical diagnosis of dementia of the Alzheimer type. Journal of Gerontology: Psychological Sciences, 60B, P98-P101.

Basso, M., Yang, J., Warren, L., MacAvoy, M.G., Varma, P., Bronen, R.A., \& van Dyck, C.H. (2006). Volumetry of amygdala and hippocampus and memory performance in Alzheimer's disease. Psychiatry Research Neuroimaging, 146, 251-261.

Celone, K.A., Calhoun, V.D., Dickerson, B.C., Atri, A., Chua, E.F., \& Millere, S.L., et al. (2006). Alternations in memory networks in mild cognitive impairment and Alzheimer's disease: An independent component analysis. Journal of Neuroscience, 26, 1022210231.

Chen, P., Ratcliff, G., Belle, S.H., Cauley, J.A., DeKosky, S.T., \& Ganguli, M. (2001). Patterns of cognitive decline in presymptomatic Alzheimer disease. Archives of General Psychiatry, 58, 853-858.

Elias, M., Beiser, A., Wolf, P.A., Au, R., White, R.F., \& D'Agostino, R.B. (2000). The preclinical phase of Alzheimer's disease: A 22-year prospective of the Framingham cohort. Archives of Neurology, 57, 808-813.

Galvin, J.E., Powlishta, K.K., Wilkins, K., McKeel, Jr., D.W., Xiong, C., Grant, E.A., et al. (2005). Predictors of preclinical Alzheimer disease and dementia: A clinicopathologic study. Archives of Neurology, 62, 758-765.

Hamann, S., Monarch, E.S., \& Goldstein, F.C. (2002). Impaired fear conditioning in Alzheimer's disease. Neuropsychologia, 40, 11871195.

Koenig, P., Smith, E.E., Moore, P., Glosser, G., \& Grossman, M. (2007). Categorization of novel animals by patients with Alzheimer's disease. Neuropsychology, 21, 193-206.

McDonald, R.J., Devan, B.D., \& Hong, N.S. (2004). Multiple memory systems: The power of interactions. Neurobiology of Learning and Memory, 82, 333-346.

Mintun, M.A., LaRossa, G.N., Sheline, Y.I., Dence, C.S., Lee, S.Y., Mach, R.H., et al. (2006). [ $\left.{ }^{11} \mathrm{C}\right] \mathrm{PIB}$ in a nondemented population: Potential antecedent marker of Alzheimer disease. Neurology, 67, 446-452.

Raichle, M.E., MacLeod, A.M., Snyder, A.Z., Powers, W.J., Gusnard, D.A., \& Shulman, G.L. (2001). A default mode of brain function. Proceedings of the National Academy of Sciences of the United States of America, 98, 676-682.

Rapp, M.A., \& Reischies, F.M. (2005). Attention and executive control predict Alzheimer disease in late life. American Journal of Geriatric Psychiatry, 13, 134-141. 
Smith-Gamble, V., Baiyewu, L., Perkins, A.J., Gurejje, O., Hall, K.S., Ogunniyi, A., et al. (2002). American Journal of Geriatric Psychiatry, 10, 724-732.

Storandt, M., Grant, E.A., Miller, J.P., \& Morris, J.C. (2006). Longitudinal course and neuropathological outcomes in original vs. revised MCI and in preMCI. Neurology, 67, 467-473.
Twamley, E.W., Ropacki, S.A.L., \& Bondi, M.W. (2006). Neuropsychological and neuroimaging changes in preclinical Alzheimer's disease. Journal of the International Neuropsychological Society, $12,707-735$. 